\title{
GENETIC POLYMORPHISM OF HUMAN RED CELL GLUTAMIC-PYRUVIC TRANSAMINASE IN AN ISOLATED COMMUNITY IN WESTERN JAPAN ${ }^{1}$
}

\author{
Itsuro NishIGAKI, ${ }^{*}$ Haruo SUZUKI, ${ }^{*}$ Norio FUIIKI, * \\ and Tetsuro NAKAI** \\ *Department of Epidemiology and of Genetics, Institute \\ for Developmental Research, Kasugai, Japan \\ **Department of Internal Medicine, Kyoto Prefectural \\ University of Medicine, Kyoto, Japan
}

\begin{abstract}
Summary The distribution of three common phenotypes of the human red cell soluble GPT has been determined in 145 inhabitants of an isolated community in western Japan. The frequency of two common alleles, $G p t^{1}=0.528$ and $G p t^{2}=0.472$, revealed somewhat different values, as compared with those of neighbouring populations. In addition, quantitative differences between the three common phenotypes of the red cell GPT has been demonstrated.
\end{abstract}

\section{INTRODUCTION}

Glutamic-pyruvic transaminase [GPT: EC 2.6.1.2] catalysing the interconversion of L-alanine and $\alpha$-ketoglutarate to L-glutamate and pyruvate exists widely in human organs, particularly plentiful in the liver. GPT exists in two molecular forms; one is mitochondrial (mGPT) and the other is cytoplasmic or soluble (sGPT).

Chen and Giblett (1971) reported that human red cell sGPT exhibited genetic polymorphism with three common phenotypes of two codominant alleles, Gpt ${ }^{1}$ and $G p t^{2}$.

Many authors have reported data on GPT gene frequencies in various populations, and so far, the $G p t^{1}$ gene frequency has been found to be higher among individuals of African, Papuan and Australian origins than among those of other ethnic groups (Chen and Giblett, 1971; Kömpf, 1971; Chen et al., 1972; Olaisen and Teisberg, 1972; Brinkmann et al., 1972; Seth, 1974; Olaisen, 1975; Welch et al., 1975; Blake, 1976).

In addition to these common alleles, several rare alleles have been detected in various populations: $G p t^{3}, G p t^{4}, G p t^{5}, G p t^{6}, G p t^{7}, G p t^{8}$ and silent allele $G p t^{0}$ (Chen

${ }^{1}$ Supported in part by a grant from the Ministry of Education, Science and Culture of Japan. Received December 12, 1977 
et al., 1972; Olaisen, 1973a: Spielmann et al., 1973; Olaisen, 1973b; McAlpine et al., 1974; Santachiara Benerecetti et al., 1975).

Ishimoto and Kuwata (1974) also reported that the $G p t^{1}$ gene frequency seemed to vary among groups tested in Japanese populations and to decrease gradually towards the southern localities in western Japan.

In this paper, we report the distribution of the GPT gene frequency in an isolated community, Sagishima, and on its quantitative differences between the three common phenotypes, for which no data in Japanese samples heretofore been presented.

\section{MATERIALS AND METHODS}

Blood samples from 145 adults were collected in the course of a medical field survey in the isolated island community, Sagishima, Hiroshima prefecture in western Japan.

The specimens, collected into $\mathrm{ACD}$ solution, were stored at $-70^{\circ} \mathrm{C}$ with an equal volume of glycerol solution, after washing. GPT typing and quantitative enzyme assay were performed immediately after hemolysis of packed red cells.

Electrophoretic separation was carried out according to a modified version of the method described by Brinkmann et al. (1972). The bridge buffer was $0.1 \mathrm{M}$ Trismalate-EDTA adjusted to $\mathrm{pH} 7.4$ with sodium hydroxide. Twelve percents starch gels (hydrolysed starch, Connaught Medical Research Laboratories) were prepared using a 1 in 15 dilution of the bridge buffer. Horizontal starch gel electrophoresis was carried out for $18 \mathrm{hr}$ at $8-9 \mathrm{~V} / \mathrm{cm}$ in a cold room. The gels were sliced and the anodal portions were stained for GPT. A Whatmann No. 3 filter paper soaked with the staining solution was carefully placed on the cut-surface of sliced gel. The gel was incubated at $37^{\circ} \mathrm{C}$ for $3 \mathrm{hr}$ and photographed with a yellow filter under long-wave ultraviolet light. The position of GPT isoenzymes was indicated by the presence of dark areas, due to the change from fluorescent NADH to non-fluorescent NAD.

The red cell GPT activity was measured by the method described by Chen $e$ al. (1972) with several modifications. The enzyme assay was performed at $37^{\circ} \mathrm{C}$ in a $0.5 \mathrm{ml}$ of reaction mixture, consisting of $0.25 \mathrm{ml}$ substrate solution and $0.25 \mathrm{ml}$ hemolysate. In this procedure, following reagents were used; substrate solution $(100 \mathrm{~mm}$ L-alanine and $35 \mathrm{~mm} \alpha$-ketoglutarate dissolved in $0.1 \mathrm{M}$ Tris- $\mathrm{HCl}$ buffer and adjusted to $\mathrm{pH} 7.8$ with sodium hydroxide), color reagent $(0.1 \% 2,4$-dinitrophenylhydrazine in $20 \% \mathrm{HCl}$ ), alkali solution $(1.25 \% \mathrm{KOH}$ in $95 \%$ ethanol) and acid solution (50\% TCA in distilled water).

As shown in Fig. 1, the absorption spectrum of 2,4-dinitrophenylhydrazone with pyruvate had a peak at $440 \mathrm{~nm}$ and a shoulder at $510 \mathrm{~nm}$. The optical density at these two wavelengths increased linearly with the amount of pyruvate from 0 to $0.75 \mu$ mole $/ 0.5 \mathrm{ml}$. Using the relationship at $510 \mathrm{~nm}$, the amount of pyruvate formed with GPT activity was determined for each sample. One unit of GPT 


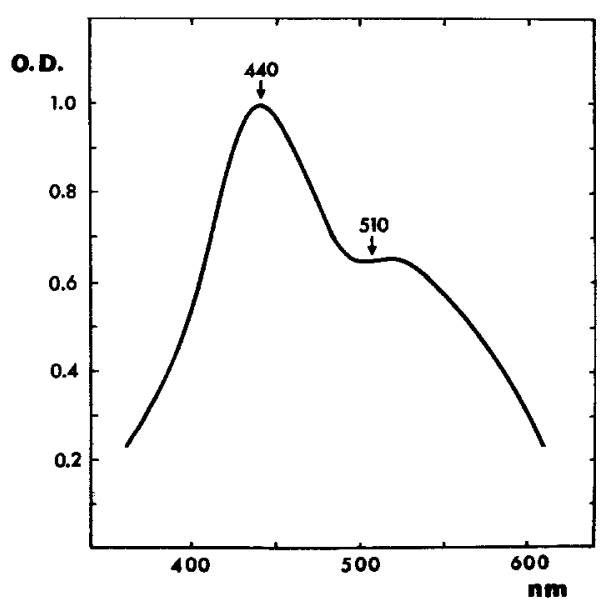

Fig. 1. Absorption spectrum of 2,4-dinitrophenylhydrazone.

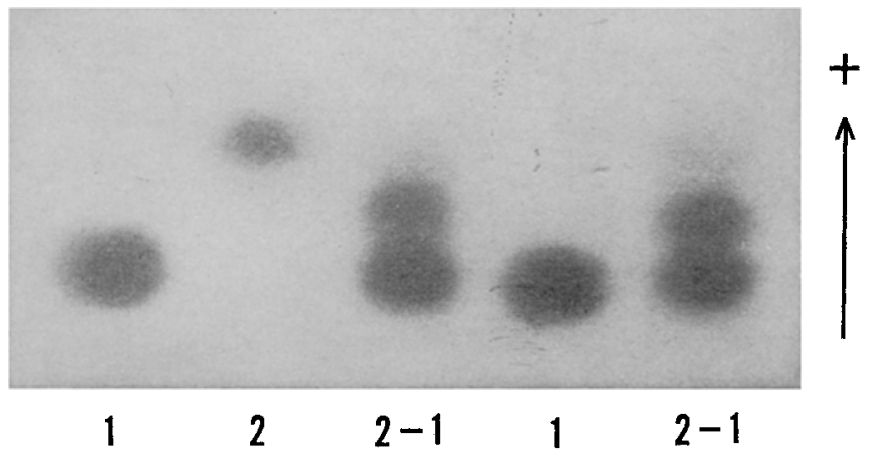

Fig. 2. Electrophoretic patterns obtained by starch gel electrophoresis at $\mathrm{pH}$ 7.4, in three common GPT phenotypes-GPT 1, GPT 2-1, GPT 2.

activity is defined as one $\mu$ mole of pyruvate formed per gram of hemoglobin per $30 \mathrm{~min}$. The amount of hemoglobin in hemolysates was determined by the cyanmethemoglobin method.

\section{RESULTS}

Three GPT common phenotypes (GPT 1, GPT 2-1 and GPT 2) were demonstrated as shown in Fig. 2, by the method of starch gel electrophoresis. The major bands of GPT 1 and GPT 2 were located anodally around $5.5 \mathrm{~cm}$ and $7.0 \mathrm{~cm}$ from the origin, respectively. The heterozygous GPT $2-1$ showed a triple band pattern, consisting of these two major bands and an intermediate component. These three bands were unequal in staining intensity, with the most anodal one being the weakest component. 
Table 1. Distribution of red cell GPT phenotypes in community surveyed: Sagishima.

\begin{tabular}{lcccc}
\hline & \multicolumn{5}{c}{ Phenotype } \\
\cline { 2 - 5 } Number & 1 & $2-1$ & 2 & Total \\
\hline Observed & 45 & 63 & 37 & 145 \\
Percentage & 31.0 & 43.5 & 25.5 & 100.0 \\
Expected & 40.4 & 72.3 & 32.3 & 145.0 \\
\hline Gene frequency & \multicolumn{5}{c}{$\mathrm{Gpt}^{1}=0.528(0.612)$} \\
& $\mathrm{Gpt}^{2}=0.472(0.388)$ \\
\hline$\chi^{2}, \mathrm{df}$ & $\chi^{2}=2.4039 ;$ & $\mathrm{df}=1 ;$ & $0.25>\mathrm{p}>0.10$ \\
\hline
\end{tabular}

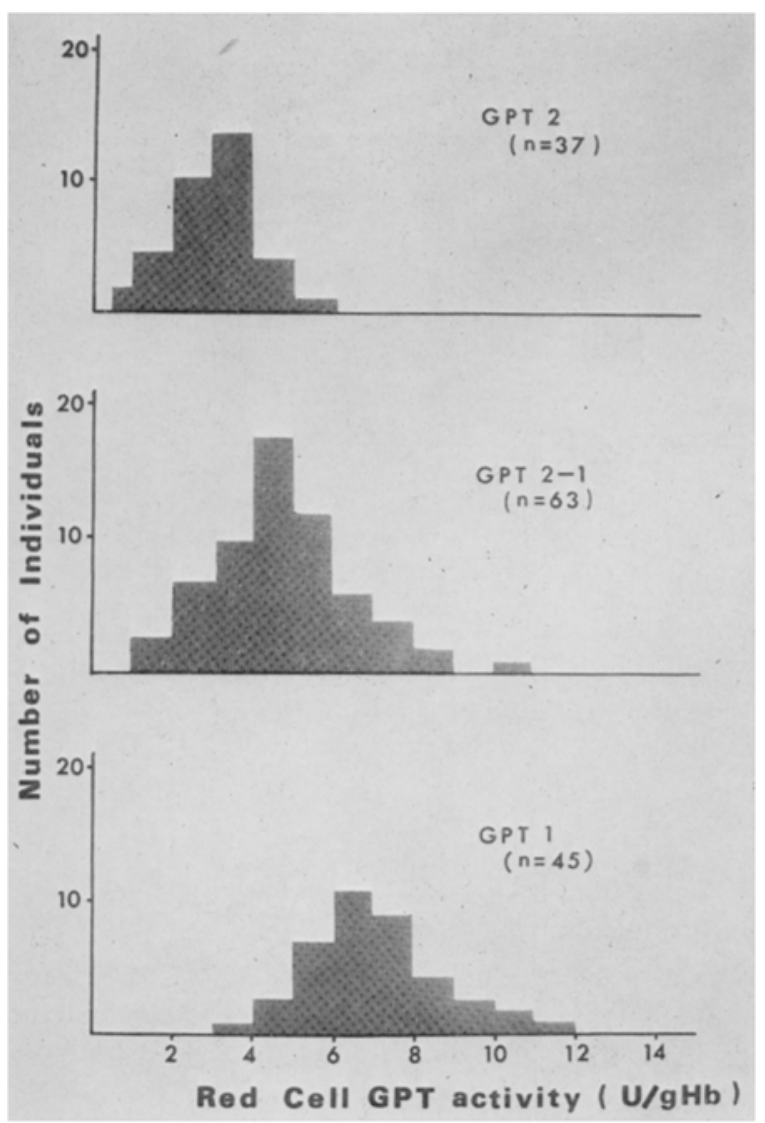

Fig. 3. Distribution of red cell GPT activities among 145 individuals, classified by three different phenotypes. 
The result collected on Sagishima survey are summarized in Table 1. The $G p t^{1}$ gene frequency in this isolated community was 0.528 , compared with that of 0.612 for a neighbouring population studied by Ishimoto et al. The gene frequencies of this and other genetic markers in this community were differed slightly from those in neighbouring populations, probably due to the highly inbred structure and small sample size of this community.

The mean activities of the three common phenotypes were found to be 6.55 units/gHb for GPT 1, 4.66 for GPT 2-1 and 2.78 for GPT 2. The distribution of GPT activity classified by the three different phenotypes is shown in Fig. 3. Thus, the highest values were found in homozygous GPT 1 individuals, the lowest values in homozygous GPT 2 individuals and the values of heterozygous GPT 2-1 individuals fall between these two levels. Chen et al. (1972) suggested that, as an average, the $G p t^{1}$ gene product in the red cell has catalytic activity about three times higher than that of $G p t^{2}$. Our results also support this suggestion.

\section{DISCUSSION}

Since it was shown by Chen and Giblett (1971) that the red cell GPT constituted a genetic polymorphism, the gene frequencies in various certain groups have been reported from various population studies. Ishimoto has also demonstrated (1975) that there might be differences among regional groups of Japanese population and that there appears to be a geographical cline, ranging from 0.624 (Mie) to 0.535 (Ishigaki Island).

It is well known that consanguinity studies provide an excellent approach to elucidate the genetic constitution of some human populations. We have already investigated the status of consanguinity in the individuals of isolated communities and its effect on genetic polymorphisms of the red cell enzymes and serum proteins (Yamamoto et al., 1972). In these isolated communities there are somewhat different gene frequencies of genetic markers, compared with those of neighbouring populations, due to probably higher consanguinity rate and mean inbreeding coeffcients. We are inclined to ascribe these differences not only to higher inbred structure but also to genetic drift and small sample size. Moreover, these isolated communities, with higher homozygosity rates, can provide us with very useful data on unusual variants. On the isolated island of Sagishima, the $G p t^{1}$ gene frequency was found to be different, in comparison with data obtained from neighbouring populations.

Quantitative differences were demonstrated in all three common phenotypes seen in this community. The values for enzyme activity of each phenotype varied insignificantly from those previously reported (Chen et al., 1972; Olaisen, 1973; Welch, 1972; 1975). However, these results are compatible with the finding that the red cell $G p t^{1}$ gene product has catalytic activity about 3 times higher than that of $G p t^{2}$. 
In this community, we did not find any evidence for the presence of a rare electrophoretic variant; however we found two cases with very low enzyme activity (0.76 and 0.89) in the GPT 2 phenotype. The existence of a silent allele Gpt ${ }^{0}$ which would show no or very weak enzyme activity was reported through the method of pedigree survey by Olaisen (1973b) and Spielmann et al. (1973). Though no convincing evidence for the presence of a silent allele could be obtained through family studies in these two cases, we also suggest there might be a heterozygote for the silent allele in the cases exhibiting a mean value of about one half normal enzyme activity.

Acknowledgements The authors express gratitude to Mr. T. Itoh for helpful technical assistance.

\section{REFERENCES}

Blake, N. M. 1976. Glutamic pyruvic transaminase and esterase D types in the Asian-Pacific area. Human Genet. 35: 91-102.

Brinkmann, B., Krukenberg, P., Brinkmann, M. and Hoppe, H. H. 1972. Gene frequencies of soluble glutamic-pyruvic-transaminase in a Northern German population (Hamburg). Humangenetik 16: 355-356.

Chen, S. H. and Giblett, E. R. 1971. Polymorphism of soluble glutamic-pyruvic transaminase: a new genetic marker in man. Science 173: 148-149.

Chen, S. H., Giblett, E. R., Anderson, J. E. and Fossum, B.L.G. 1972. Genetics of glutamicpyruvic transaminase: its inheritance, common and rare variants, population distribution, and differences in catalytic activity. Ann. Human Genet. 35: 401-409.

Ishimoto, G. and Kuwata, M. 1974. Red cell glutamic-pyruvic transaminase polymorphism in Japanese populations. Jap. J. Human Genet. 18: 373-377.

Ishimoto, G. 1975. Red cell enzyme. In Anthropological and Genetic Studies on the Japanese (S. Watanabe, S. Kondo and E. Matsunaga, eds.), Chap. 3, Tokyo Univ. Press, Tokyo, p. 109.

Kömpf, J. 1971. Population genetics of soluble glutamic-pyruvic transaminase [EC: 2.6.1.2]: gene frequencies in Southwestern Germany. Humangenetik 14: 76-77.

McAlpine, P. J., Chen, S. H., Cox, D. W., Dossetor, J. B., Giblett, E. R., Steinberg, A. G. and Simpson, N. E. 1974. Genetic markers in blood in a Canadian Eskimo population with a comparison of allele frequencies in Circumpolar populations. Human Hered. 24: 114-142.

Olaisen, B. and Teisberg, P. 1972. Erythrocyte alanine aminotransferase polymorphism in Norwegian Lapps. Human Hered. 22: 380-386.

Olaisen, B. 1973a. Two rare GPT phenotypes in a Norwegian family: evidence of seventh allele. Humangenetik 19: 289-291.

Olaisen, B. 1973b. Atypical segregation of erythrocyte glutamic-pyruvic transaminase in a Norwegian family. Human Hered. 23: 595-602.

Olaisen, B. 1975. Distribution of GPT types in Norway. Human Hered. 25: 20-29.

Santachiara Benerecetti, A. B., Beretta, M. and Pampiglione, S. 1975. Red cell glutamic-pyruvic transaminase polymorphism in a sample of the Italian population. A new variant allele: GPT8. Human Hered. 25: 276-278.

Seth, S. 1974. Population genetics of soluble glutamic-pyruvic-transaminase in North Germans (Lübeck). Humangenetik 23: 223-226.

Spielmann, W., Kühnl, P., Rexrodt, Ch. und Hänsel, G. 1973. Untersuchungen zum GPT-System unter besonderer Berücksichtigung des stummen Allels GPT ${ }^{0}$. Humangenetik 18: 341-348.

Welch, S. G. 1972. Quantitative differences between the human red cell glutamate-pyruvate transaminase phenotypes. Human Hered. 22: 190-197. 
Welch, S. G., Mills, P. R. and Gaensslen, R. E. 1975. Phenotypic distributions of red cell glutamatepyruvate transaminase [E.C. 2.6.1.2] isoenzymes in British and New York populations. Humangenetik 27: 59-62.

Welch, S. 1975. Comparative studies on the human glutamate-pyruvate transaminase phenotypesGPT 1, GPT 2-1, GPT 2. Humangenetik 30: 237-249.

Yamamoto, M., Wada, T., Watanabe, T., Kanazawa, H., Saito, R., Kondo, M., Hosokawa, K., Masuda, M., Nakai, T. and Fujiki, N. 1972. Genetic polymorphisms in! four isolated communities in Kinki district. Jap. J. Human Genet. 17: 273-285. 